\title{
The Potential Power of Bar-HRM Technology in Herbal Medicine Identification
}

\author{
Wei Sun ${ }^{1 \dagger}$, Jing-jian $\mathrm{Li}^{1,2+}$, Chao Xiong ${ }^{1}, \mathrm{Bo}$ Zhao ${ }^{1,3}$ and Shi-lin Chen ${ }^{1 *}$ \\ ${ }^{1}$ Institute of Chinese Materia Medica, China Academy of Chinese Medical Sciences, Beijing, China, ${ }^{2}$ College of Forestry and \\ Landscape Architecture, South China Agricultural University, Guangzhou, China, ${ }^{3}$ Zhuhai College of Jilin University, Zhuhai, \\ China
}

The substitution of low-cost or adulterated herbal products for high-priced herbs makes it important to be able to identify and trace herbal plant species and their processed products in the drug supply chain. PCR-based methods play an increasing role in monitoring the safety of herbal medicines by detecting adulteration. Recent studies have shown the potential of DNA barcoding combined with high resolution melting (Bar-HRM) analysis in herbal medicine identification. This method involves precisely monitoring the change in fluorescence caused by the release of an intercalating DNA dye from a DNA duplex as it is denatured by a gradual increase in temperature. Since the melting profile depends on the GC content, length, and strand complementarity of the amplification product, Bar-HRM analysis opens up the possibility of detecting single-base variants or species-specific differences in a short region of DNA. This review summarizes key factors affecting Bar-HRM analysis and describes how Bar-HRM is performed. We then discuss advances in Bar-HRM analysis of medicinal plant ingredients (herbal materia medica) as a contribution toward safe and effective herbal medicines.

Keywords: Bar-HRM technology, herbal medicine, adulteration, substitution, drug safety

\section{INTRODUCTION}

Herbal medicines have played a significant role in preventing chronic diseases and improving health for human beings since ancient times. According to the World Health Organization [WHO] (2003), over $70 \%$ of the population in developing countries uses herbal products (http://www.who. int/mediacentre/news/releases/2004/pr44/en/). The past decade has seen increasing global demand for herbal medicines despite the economic recession (GIA, 2013). Herbal medicines are clearly gaining global influence in modern medical and health services. At an international conference in February 2013, WHO Director General Dr. Margaret Chan stated that traditional medicines of proven quality, safety, and efficacy contribute to the goal of ensuring that all people have access to care (World Health Organization, 2013). Consumer safety is an issue that cannot be overlooked as the herbal industry grows. However, the large number and variety of medicinal plants currently sold in various markets, along with the fact that some of them are commonly sold in processed or modified forms (e.g., dried material, tablets, powders, and capsules), presents a challenge to efforts to accurately distinguish genuine products from their close relatives, inferior substitutes, adulterants, and counterfeits (Newmaster et al., 2013; Veldman et al., 2014). Inaccurate identification of herbal material has led to several safety-related incidents. For example, due 
to their similar appearance and Chinese names, a herbal ingredient derived from Astragalus complanatus bunge (shayanzi), which is used for diabetes treatment, was inadvertently replaced with one from Hyoscyamus niger (tianxianzi), which interrupts the parasympathetic nervous system. H. niger contains L-Hyoscyamine Atropine and Scopolamine, natural compounds derived from Hyoscyamus L. species, which can overstimulate the sympathetic nervous system and cause convulsions and, eventually, death (Li et al., 2012; Tu et al., 2014). Incidents involving the use of Radix Stephaniae tetrandrae (Fangji), derived from Stephania tetrandra S. Moore, have also been reported (Vanherweghem et al., 1993; Grollman et al., 2007; Debelle et al., 2008; Poon et al., 2013). In one case, patients were prescribed the Chinese herb Fangji (Stephania tetrandra S. Moore) for weight loss, but were instead given Guangfangji (Aristolochia fangchi Y. C. Wu ex L. D. Chow and S. M. Hwang), which contains aristolochic acids. The patients subsequently developed aristolochic acid nephropathy (Lord et al., 1999). Despite this, the marketing of herbs containing Aristolochiaceous materials continues in China (Wu et al., 2015). In addition to safety concerns, the quality of herbal products has been a subject of increasing attention. Although commercial herbal products have to be authentic as well as safe, adulterated herbal materials have been found on sale (Ouarghidi et al., 2012; Xin et al., 2015). For example, processed materials of the relatively rare herbaceous perennial Panax ginseng C. A. Mey are commonly adulterated with material from the low-cost herb Panax quinquefolius (Chen et al., 2008). Accurate identification of medicinal plant ingredients is therefore important to safeguard customer health and ensure the quality and authenticity of herbal products.

Various criteria and methods have been developed to authenticate medicinal plant ingredients. Traditional methods include the use of morphological characteristics, microscopy, and chemical profiles (Li et al., 2010; Lau et al., 2012; Shen et al., 2014), and these methods have played a major role in herbal drug authentication and quality control since the birth of pharmacognosy. However, these techniques all have limitations. In addition to possible confusion caused by morphological similarity or variation in chemical profiles between samples, the accuracy of these methods depends on the expertise of the assessor ( $\mathrm{Li}$ et al., 2011). In addition, morphological identification is often not possible when the original plant material has been heavily processed. Additional methods have therefore been sought. Advancements in molecular biology and molecular genetics have allowed the application of DNAbased methods to identify and authenticate medicinal plants. DNA barcoding combined with high resolution melting (BarHRM) analysis is a novel, advanced method which has recently been successfully applied in herbal medicine authentication (Mader et al., 2011; Bosmali et al., 2012; Xanthopoulou et al., 2016).

In this review, we introduce the principle of HRM, point out key factors affecting the analysis, and describe how HRM is performed and how the data analyzed. We then continue with a brief overview of its current role in scientific studies, and finally present and critically discuss the potential power of Bar-HRM analysis based methods in herbal medicine authentication.

\section{HIGH RESOLUTION MELTING}

\section{Overview of HRM Technology}

High Resolution Melting technology characterizes nucleic acid samples based on their disassociation behavior, using direct melting to detect small sequence differences in PCR-amplified sequences. These differences are detected through the use of DNA-specific dyes, high-end instrumentation and sophisticated analysis software. Samples are discriminated according to their composition, length, GC content, and strand complementarity (Ririe et al., 1997; Reed and Wittwer, 2004; Palais et al., 2005). The first step is standard PCR amplification of the region of interest in the presence of a specialized double-stranded DNA (dsDNA) binding dye (Wittwer et al., 1997, 2003; Herrmann et al., 2006). There are various types of dsDNA intercalating dyes, including SYBR Green, LC Green PLUS, Eva Green, SYTO9, and ResoLight. SYBR Green is the most common non-saturating dsDNA intercalating dye. It is generally unsuitable for most HRM applications because it has been shown to inhibit PCR at high concentrations and has been hypothesized to redistribute from melted regions back into the dsDNA amplicon, as detailed in Figure 1A (von Ahsen et al., 2001; Graham et al., 2005). To overcome this limitation, a new class of dsDNA intercalating dyes named saturating or "release-on-demand" dyes that do not inhibit DNA polymerases, or alter the Tm of the product, have recently been developed. In contrast to SYBR Green, Saturating dyes such as SYTO9 or LCGreen do not inhibit the DNA polymerase at rather high concentrations, ensuring more complete intercalation of the amplicon. More precise examination of the melting behavior is therefore possible for HRM analysis, as indicated in Figure 1B (Vossen et al., 2009). The "release-on-demand" dyes, e.g., EvaGreen, can be used at non-saturating concentrations. Due to its novel mechanism of fluorescence emission, the fluorescent signal is quenched when the dye is free in solution. Instead, the dye emits high fluorescent signal when it binding to duplex DNA (Figure 1C). There is no PCR inhibition, whilst the unique dye provides highly sensitive HRM analysis.

As mentioned above, the saturating or "release-on-demand" dyes have high fluorescence when bound to dsDNA and low fluorescence when unbound, allowing the user to monitor DNA amplification during PCR (Figure 2A). HRM analysis begins after PCR amplification. The amplicon is gradually heated from around $50^{\circ} \mathrm{C}$ to around $95^{\circ} \mathrm{C}$; the gradual denaturation resulting from incremental heating produces a characteristic melting profile (Wittwer et al., 2003). When the dsDNA dissociates into single strands, the intercalating dye is released and fluoresces at a low level. The change in fluorescence is plotted against the temperature, generating a melting curve (Figure 2B) characteristic of the amplicon. The melting temperature (Tm) of the amplicon can be determined from the peak obtained by plotting the negative derivative of the fluorescence $(\mathrm{F})$ over temperature $(\mathrm{T})(-\mathrm{dF} / \mathrm{dT})$ against the temperature (T; Figure $2 \mathrm{C}$ ) 


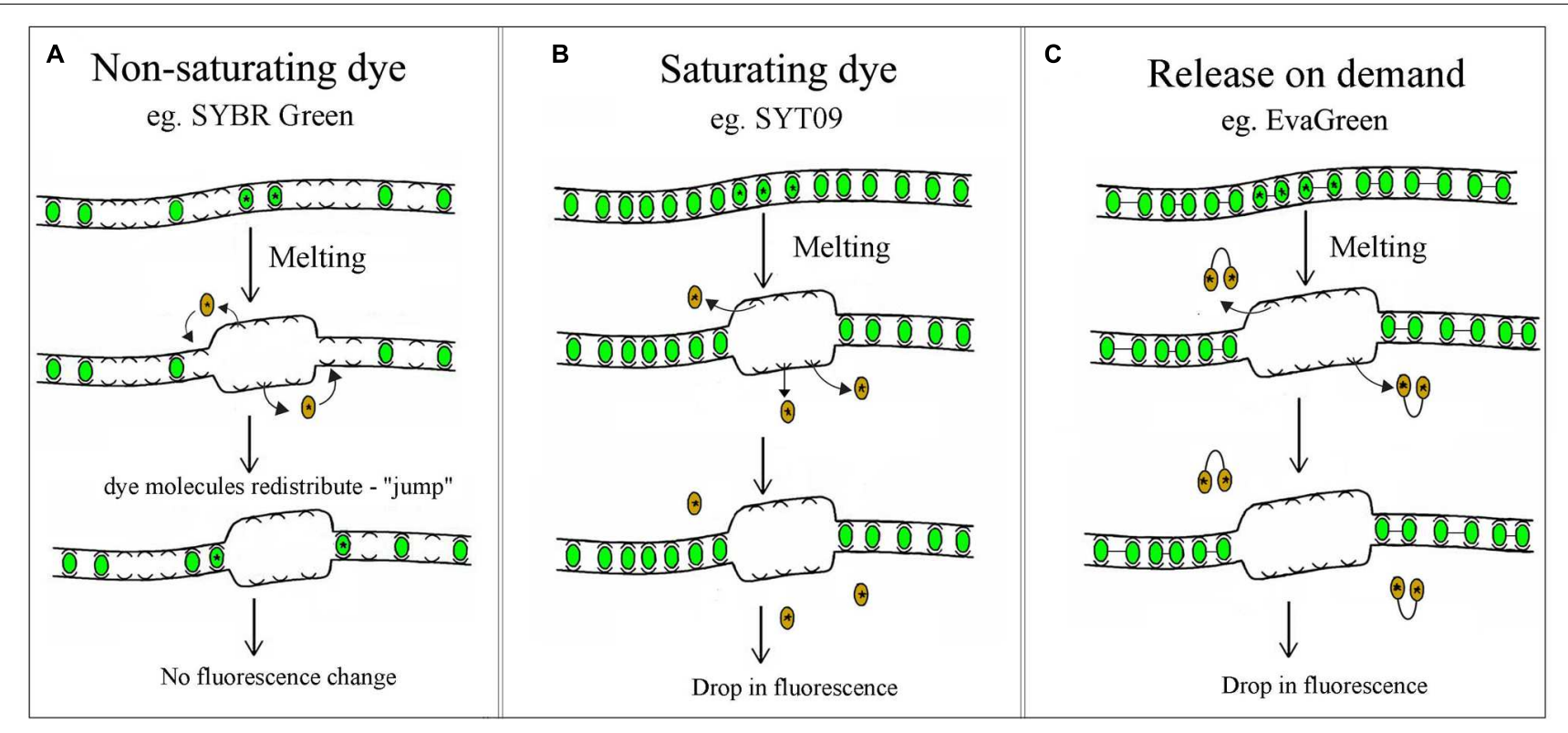

FIGURE 1 | Non-saturating, saturating and "release-on-demand" dsDNA intercalating dyes. (A) At non-saturating concentrations the dye quickly rebinds to regions that remain double stranded, consequently there is no drop in fluorescence. (B,C) Saturating and "release-on-demand" dyes do not redistribute from the melted regions of single-stranded DNA back to dsDNA, resulting in a reduction of fluorescence. This difference gives dyes such as SYT09 or EvaGreen the high sensitivity required for HRM analysis.

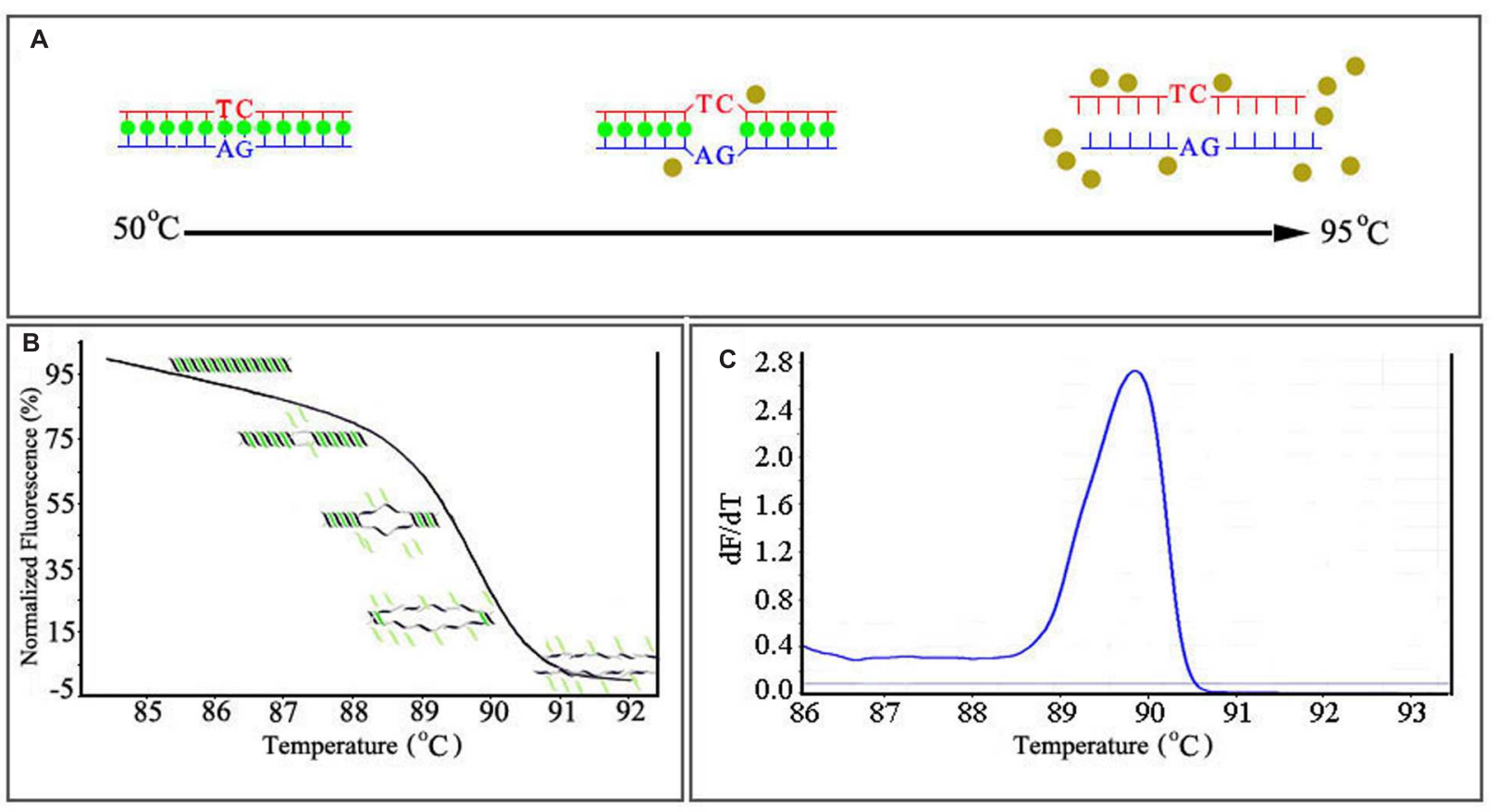

FIGURE 2 | The mechanism of real-time HRM system. (A) The dissociation-characteristics (Melting) of double-stranded DNA during heating. (B) The normalized plot generated by high resolution melting (HRM) analysis. High fluorescence when dye is bound to dsDNA. (C) The melting curves (derivative melt curves) of the amplicons, negative derivative of the fluorescence (F) over temperature $(T)(-d F / d T)$ against the temperature $(T)$.

(Montgomery et al., 2007). Since different genetic sequences melt at slightly different rates, they can be viewed, compared, and detected using these curves. When correctly set up, HRM is sensitive enough to allow the detection of a single base change between otherwise identical nucleotide sequences (Wittwer et al., 2003; Zhou et al., 2005; Krypuy et al., 2006). 


\section{Key Factors Affecting HRM Assays}

For successful analysis, care should be taken to ensure that the experiment is optimized for HRM. Particular attention should be given to primer design, PCR reagents and cycling conditions, since small differences in melting curves can arise from sources other than the nucleotide sequence. Factors such as genomic DNA (gDNA) quality, amplicon length, primer design, dye selection, and PCR conditions will all affect the melting behavior (Montgomery et al., 2007; van der Stoep et al., 2009). Achieving specific amplification is critical to the success of the assay, since any non-specific amplification will greatly impair the melt analysis. For example, a low-quality DNA template may produce non-specific PCR products, resulting in failed reactions or low sensitivity and incorrect genotype calls. Samples that amplify late or fail to reach a high signal plateau in the PCR phase can lead to inconclusive or low-resolution HRM data. For the best results, all DNA samples in an analysis run should be prepared using the same method. DNA samples should not differ significantly in their concentration in order to ensure similar cycle threshold (Ct) values (Reed et al., 2007; Vossen et al., 2009). Furthermore, HRM analysis should be performed immediately following PCR whenever possible. An amplicon length of 50-300 bp is generally recommended for HRM analysis of different sequence variants, including single nucleotide polymorphisms (SNPs), inversions, insertions, and deletions. The longer the amplicon, the more difficult it is to clearly discriminate between sequence variants. However, longer amplicons (typically 200-500 bp) can be used when screening for unknown sequence differences (Montgomery et al., 2007, 2010; Erali et al., 2008). This is useful in gene scanning or determining the variation within a population. Primers designed for HRM analysis should be optimized for robust performance and specificity to the region of interest, since the dyes will bind to any dsDNA products. In addition, HRM software may not be able to detect non-specific reaction products if their melting profiles are similar. It is therefore best to initially assess PCR products by agarose gel electrophoresis (Wittwer, 2009). Other important factors are the PCR conditions and the choice of intercalating dye. The PCR conditions should be optimized in order to achieve efficient amplification, indicated by low $\mathrm{Ct}$-values and amplification curves plateauing (Vossen et al., 2009). Several different HRM dyes should be tested to determine which functions best in a given experimental system. One factor affecting dye functionality is the instrument itself, since different instruments use different detection methods.

\section{HRM Data Analysis}

With advanced software tools, data analysis is typically straightforward, and multiple samples can be analyzed simultaneously. However, it is important to know what to look for when analyzing raw HRM melting profiles. The raw data collected during HRM analysis includes fluorescence readings across a range of temperatures which consists of three parts: pre-melt, melt, and post-melt (Figure 3A). The variance makes it difficult to properly analyze the results even though different genotype groups may be visible. The parallel double-bars in the interface should be positioned to select pre- and post-melt regions with which to normalize data (Figure 3A). If the preor post-melt regions cannot be clearly identified, the HRM run should be repeated with the temperature range adjusted as required.

When the data is normalized correctly, it will appear as shown in Figure 3B. This is termed "Normalization Data." In this plot, the fluorescence variance of the pre- or post-melt regions has been removed, and only the temperature range between the outer bars of the pre- and post-melt regions is shown. The genotypes are now more distinct, but the differences between melting curves are often small in some cases. In order to better visualize small differences between individual melting curves, some HRM software applications allow calculation of a difference plot (Figure 3C). In this plot, sample curves are subtracted from a single reference run in the same experiment. Any genotype can be selected as the reference, but typically a wild-type control is used; in the case of species identification, genotypes of test samples can be defined by selecting a representative sample for each species. In addition, a genotype confidence percentage (GCP; the confidence that a sample is the same as the reference genotype, with a value of 100 indicating an exact match) is calculated for each genotype by some HRM software. Depending on the study's objective, the confidence threshold can be adjusted to group the samples. However, it is advisable to confirm the genotypes by checking the difference plot.

\section{BAR-HRM METHOD TO IDENTIFY AND CERTIFY HERBAL MATERIAL}

High resolution melting analysis has several advantages over traditional methods for gene scanning and genotyping. The analysis is performed immediately after amplification and it is not necessary to purify or separate the amplicons, making HRM analysis particularly suitable for medium- to high-throughput amplification. In addition, HRM analysis is less expensive than other approaches, such as DNA sequencing. Its ease of use, flexibility, low cost, superb sensitivity, and specificity have led to the adoption of HRM for clinical research and diagnostics, including the detection of cancer-specific mutations (Akiyoshi et al., 2013; Lin et al., 2014) and DNA methylation (Wojdacz, 2012; Wojdacz et al., 2014), as well as for the authentication of food products (Ganopoulos et al., 2012; Madesis et al., 2012; Sakaridis et al., 2013) and the accurate quantitation and detection of bacteria (Duyvejonck et al., 2015). Very recently, a new method combining DNA barcoding with HRM analysis (BarHRM) was developed for the authentication of herbal medicines and for accurate quantitation of adulterants in commercial herbal medicine products. In the following section, we critically discuss the application of Bar-HRM methods in herbal medicine identification and provide an overview of developing a Bar-HRM assay.

\section{DNA Barcoding}

DNA barcoding is a technique used to identify species based on a short, standardized fragment of the genome called "DNA barcode" (Hebert et al., 2003). This short sequence of 


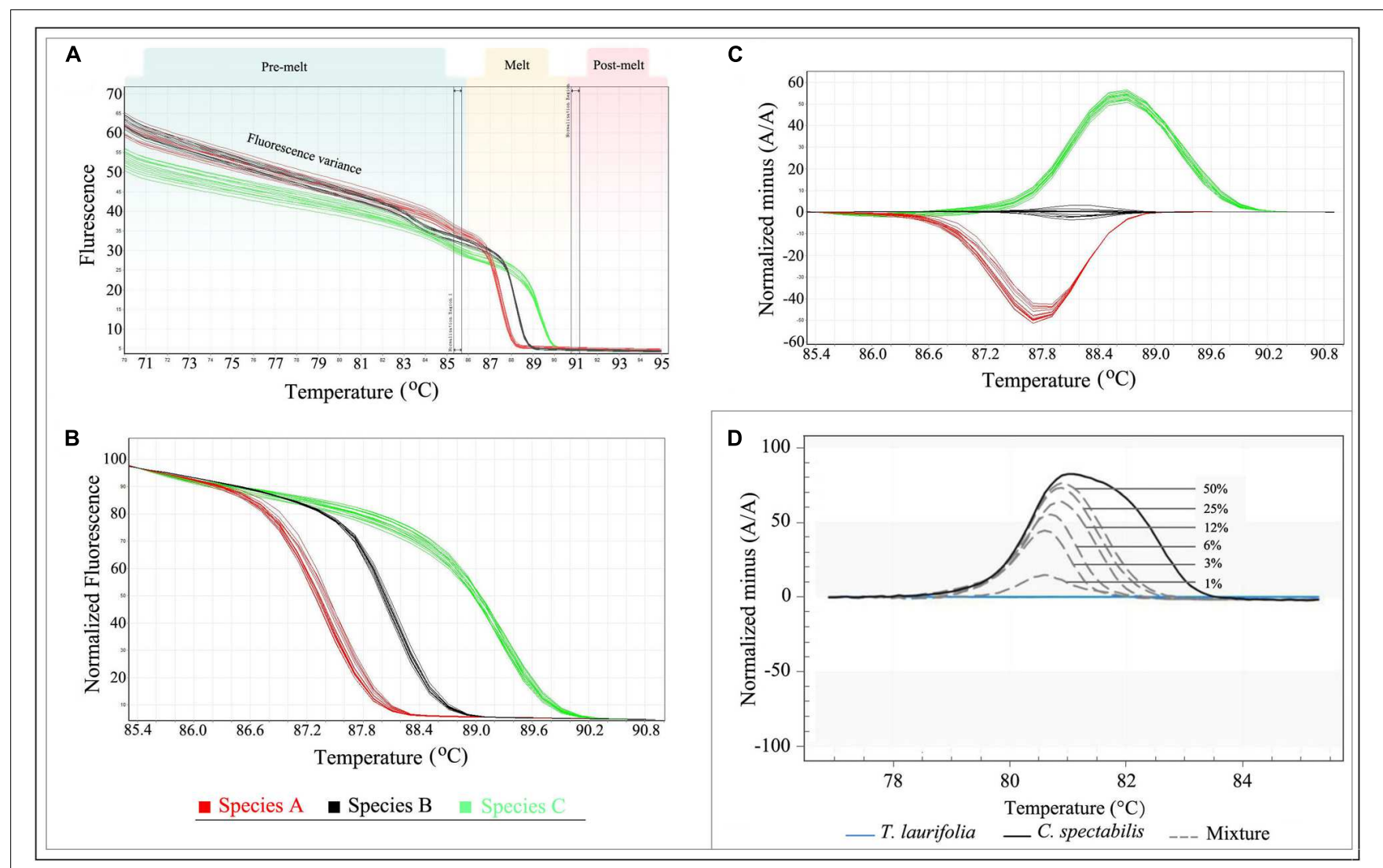

FIGURE 3 | Analysis of HRM data. (A) Raw Data Melt Curve. (B) Normalization Data derived from the raw data plots in (A). (C) Difference Graph derived from the Normalization Data. (D) Specific amplicons and applied to reference mixtures containing 50, 25, 12, 6, 3, and 1\% of C. spectabilis in T. laurifolia. Note: Figure 3D adopted from reference (Singtonat and Osathanunkul, 2015).

nucleotides could be from an appropriate part of the chloroplast, mitochondrial, or nuclear genome and is used to identify organisms at the species level (Ryan, 2005; Miller, 2007). The mitochondrial coxI gene (COI) was suggested as a standard DNA barcode region for metazoans, and is now well established for most animal species identification (Hebert et al., 2003, 2004; Ward et al., 2005; Galimberti et al., 2012). However, the COI gene from plants have limited usefulness for identifying plant species due to the low amounts of variation in the genes, and shows intra-molecular recombination (Mower et al., 2007; Fazekas et al., 2008). Thus, screening for an analogous of COI gene in plants has focused on the nuclear and chloroplast genomes. Recent studies suggested that the internal transcribed spacer (ITS) of nuclear ribosomal DNA could be potential barcodes (Chase et al., 2005; Kress et al., 2005). In addition, several plastidial genes, such as the $r p o \mathrm{C} 1, r b c \mathrm{~L}, m a t \mathrm{~K}, t r n \mathrm{H}-p s b \mathrm{~A}$ and $p s b \mathrm{~K}-p s b \mathrm{I}$ have also been proposed as barcode regions, because of their fast evolution rate (Kress et al., 2005; Fazekas et al., 2008). For example, Kress et al. (2005) compared 10 loci for authenticating closely related species in seven plant families and 99 species belonging to 88 genera in 53 families, and they reported that the psbA-trn $\mathrm{H}$ and ITS regions could be used as a pair of potential barcodes for identifying widely divergent angiosperm taxa (Kress et al., 2005). However, this pair failed to distinguish members of the order Cycadales (Sass et al., 2007). The Consortium for the Barcode of Life (CBOL) Plant Working Group (2009) suggested to combine $r b c \mathrm{~L}$ and $m a t \mathrm{~K}$ as core-barcode regions, because of the straightforward recovery rate of $r b c \mathrm{~L}$, and the high resolution of $m a t \mathrm{~K}$. Unfortunately, mat $\mathrm{K}$ is clearly lacking the feature of universal amplification (Dunning and Savolainen, 2010). In another study, two separate regions ( $p s b \mathrm{~A}-\operatorname{trn} \mathrm{H}$ and $r b c \mathrm{~L}$ ) had significant discrimination efficiency and correctly discriminate $88 \%$ of 96 diverse species of 48 genera from 43 families (Kress and Erickson, 2007). Altogether, although no single plant marker has been found that works as well as the COI in animals, several markers such as ITS, $p s b \mathrm{~A}-t r n \mathrm{H}, m a t \mathrm{~K}, r b c \mathrm{~L}$, and $r p o \mathrm{Cl}$, have shown their superior qualities as core DNA barcodes.

Chen et al. (2010) compared seven candidate DNA barcodes $(p s b \mathrm{~A}-\operatorname{trn} \mathrm{H}, \operatorname{mar} t \mathrm{~K}, r b c \mathrm{~L}, r p o \mathrm{C} 1, y c f 5$, ITS2, and ITS) in more than 6,600 medicinal plant samples belonging to 4,800 species from 753 distinct genera. They found that the ITS2 of nuclear ribosomal DNA represents the most suitable region for DNA barcoding applications and that the rate of successful identification with ITS2 is $92.7 \%$ at the species level (Chen et al., 2010). Since then, the ITS2 region has been shown to be able to discriminate a wide range of medicinal plants (Sun et al., 2011; Pang et al., 2013; Zomuanpuii et al., 2013; Wu et al., 2015). Another three regions, $p s b \mathrm{~A}-t r n \mathrm{H}, m a t \mathrm{~K}$, and $r b c \mathrm{~L}$, 
have also been proposed as markers for barcoding medicinal plants (Liu et al., 2011; Mahadani and Ghosh, 2013; Nair et al., 2013). Although there is still debate on the effectiveness of DNA barcoding, this technology can achieve rapid, accurate, and automated identification of species from a diverse range and quality of raw materials. This addresses the difficulties involved in classifying herbal materials and promises to fuel a taxonomic renaissance in herbal identification.

\section{Bar-HRM is a Powerful Tool for Herbal Medicine Industry and Market}

In markets, herbal products are commonly sold without packaging or labels, leading to a high risk of acquiring counterfeited, substituted and/or adulterated products. Species identification is critical to ensuring quality in the herbal medicine industry. Bar-HRM has been proven to be an effective tool for determining the origin and quality of raw materials and detecting adulterations (e.g., admixture with products from other species) in the herbal processed products chain (Kalivas et al., 2014; Tong et al., 2014; Buddhachat et al., 2015; Hu et al., 2015; Osathanunkul et al., 2015a,b; Singtonat and Osathanunkul, 2015; Costa et al., 2016). This new approach has its own advantage compare with previously employed methods, an advantage of performing BarHRM analysis is that the PCR amplification and HRM analysis are performed in the one completely "closed tube" run and the results are available for analysis at the end of the run. An overview of the Bar-HRM analysis developed in herbal medicine identification is provided in Table 1.

Kalivas et al. (2014) developed an HRM based method coupled with DNA barcode ITS2 for verifying the identity of Sideritis species, evidencing Bar-HRM analysis was suitable for the identification of Sideritis species (Kalivas et al., 2014). This is the first report on the utilization of the HRM approach for rapid discrimination of Sideritis species and sparked a new line of research into possible identification for herbal medicine products. Since then, another research group has used HRM analysis method coupled with plastid DNA region psbA-trn $\mathrm{H}$ to distinguish traditional Chinese medicine Panax notoginseng from adulterant species (Tong et al., 2014), as well as the same method devoted to the identification of Chinese herbs Mutong (Akebia quinata), Chuanmutong (Clematis armandii), and Guanmutong (Aristolochia manshuriensis; $\mathrm{Hu}$ et al., 2015). These studies revealed that original species could be distinguished from adulterant species by differences in the melting curves.

Like DNA Barcoding method, the weakness of universal barcode markers was also existed in Bar-HRM analysis when identify closely related species, where genetic variability is limited. To overcome this weakness, Osathanunkul et al. (2015a) developed a minibarcoding method. By redesigning the $r b c \mathrm{~L}$ primers, researchers were able to identify and authenticate three medicinal Acanthaceae species (Acanthus ebracteatus, Andrographis paniculata, and Rhinacanthus nasutus), demonstrating the reduced length of $r b c \mathrm{~L}$ sequences could provided enough informative to identify closely related species (Osathanunkul et al., 2015a). In another study, Costa et al. (2016) pursued a very similar strategy to develop a Bar-HRM

method for the authentication of Hypericum perforatum and Hypericum androsaemum in herbal infusions. They compared the effectiveness of ITS1 and mat K minibarcode for HRM analysis, and found that mat $\mathrm{K}$ can be used as an adequate mini-barcode for the differentiation of both species.

The identification of substitutions and admixtures in herbal products is one of the more interesting applications of Bar-HRM analysis. In many cases, commercial fraud involves adulteration, whether in the substitution of low-cost herbs for high-priced ones or the fraudulent labeling of herbal products. Adulteration also raises a number of concerns regarding health (e.g., toxic substances) and diet (e.g., nutritional value). Fraud control is therefore needed in order to support fair trade and protect consumer rights. In previous studies, DNA barcoding has proven successful for detecting substitution in herbal medicines (Chen et al., 2010; Liu et al., 2011; Mahadani and Ghosh, 2013; Nair et al., 2013). However, identifying the constituent species in a mixed herbal product has proven impossible unless preceded by a cloning approach, since the DNA extracted from

TABLE 1 | Examples for application of Bar-HRM technology in herbal medicine identification.

\begin{tabular}{|c|c|c|}
\hline Application & Target DNA region & Reference \\
\hline $\begin{array}{l}\text { Discrimination of } 12 \\
\text { closely related Croton }\end{array}$ & $\begin{array}{l}\text { Internal transcribed } \\
\text { spacer } 1 \text { (ITS1) and }\end{array}$ & $\begin{array}{l}\text { Osathanunkul et al., } \\
2015 b\end{array}$ \\
\hline
\end{tabular}

species

Taxonomic identification of Sideritis species growing in Greece Differentiation of commercial Panax notoginseng from its adulterant species

Distinguish the Chinese herbs Mutong (Akebia quinata) and

Chuanmutong (Clematis armandii) from Guanmutong (Aristolochia manshuriensis)

Evaluation of Three Medicinal Products Derived from Acanthaceae Species Authentication of Hypericum perforatum and Hypericum androsaemum in herbal infusions

Detection of toxic Crotalaria spectabilis Roth. in Thunbergia laurifolia Lindl. herbal products

Authenticity analyses of Phyllanthus amarus to control its quality for medicinal plant product plastid DNA (matK, $r b c \mathrm{~L}, r p o \mathrm{C}$, and $t r n \mathrm{~L}$ ) Internal transcribed spacer 2 (ITS2)

The plastid DNA region Tong et al., 2014

psbA-trnH

The plastid DNA region psbA-trnH

Kalivas et al., 2014

Hu et al., 2015

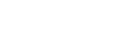


these products yields contaminated PCR-amplification products (Galimberti et al., 2013). Bar-HRM may provide an effective approach to overcome this problem, allowing the successful identification of mixed herbal products. Buddhachat et al. (2015) and Singtonat and Osathanunkul (2015) demonstrated the use of Bar-HRM analysis to assess the amount of adulterant in herbal medicine products. Singtonat and Osathanunkul (2015) evaluated four DNA barcodes $m a t \mathrm{~K}, r b c \mathrm{~L}, r p o \mathrm{C}$, and $t r n \mathrm{~L}$, and found that $r p o \mathrm{C}$ minibarcode coupled with HRM analysis was able to detect toxic herb Crotalaria spectabilis adulterants in Thunbergia laurifolia herbal products as low as 1\% (Figure 3D). Another one study reported the same limit of detection for the contamination of Phyllanthus amarus with other Phyllanthus species. The authors also highlighted the rapid detection of BarHRM. In their study, the identity of an unknown contaminant in processed commercial herbal products can be verified within $2 \mathrm{~h}$ (Buddhachat et al., 2015).

These case studies and technical advancements clearly indicate that Bar-HRM is a sensitive, fast, cheap, and reliable method for identifying and tracking a wide range of raw and processed medicinal products, as well as for detecting adulteration or poisonous components potentially occurring in commercial frauds.

\section{Developing a Bar-HRM Assay for Herbal Material}

Establishing a Bar-HRM assay for the identification of herbal material involves sample collection, verification of voucher herbarium specimens, DNA extraction, experiment optimization, HRM-PCR amplification, melting profile analysis, and finally, species identification and adulterant detection.

\section{Sample Collection and Morphological Verification}

It is crucial to be sure of the identity of the material used during the development of a Bar-HRM assay. At least three duplicate collections per species should be used; each collection (uniquely numbered) must include a small plant sample for DNA extraction and voucher herbarium specimens of the whole plant (ideally flowering or fruiting) and must be sourced from the same plant or population of plants. The collections should be accompanied by photos and detailed field notes describing any identifying characteristics not evident from the herbarium specimens. The samples must be in good condition (e.g., devoid of contamination). A detailed guide to making herbarium specimens can be found in the herbarium handbook (Bridson and Forman, 1992).

\section{DNA Extraction}

High-quality genomic DNA is an essential prerequisite for accurate identification using a Bar-HRM assay. DNA extraction from the herbal material must therefore be performed carefully and quickly, using good sterile technique to avoid DNA degradation and contamination between samples. Referred to related literatures, high quality DNA sample can be obtained by using modified CTAB method (Tel-zur et al., 1999; Chen et al., 2013, 2014). Reagents used in the method include the following: CTAB buffer containing 2\% CTAB, $100 \mathrm{mM}$
Tris-HCl, $5 \mathrm{mM}$ EDTA, $710 \mathrm{mM} \mathrm{NaCl}, 350 \mathrm{mM}$ Sorbitol, $0.1 \%$ Tris (2-carboxyethyl) phosphine hydrochloride and $1 \%$ 2-mercaptoethanol (added just before use); Washing buffer: $100 \mathrm{mM}$ Tris-HCl, $5 \mathrm{mM}$ EDTA, 5\% glycerinum, 10\% PEG8000, 0.1\% Tris (2-carboxyethyl) phosphine hydrochloride; Extraction buffer: Chloroform:Isoamyl alcohol (24:1, vol/vol); polyvinylpyrrolidone (PVP). DNA extraction procedure consists of the following stages: (1) Harvest about 50-100 mg herbal material, clean the surface with 75\% alcohol; (2) Mix the herbal material with $30 \mathrm{mg}$ PVP, grind into a fine powder under liquid nitrogen; (3) Transfer the powder into a $2.0 \mathrm{ml}$ centrifuge tube, add $1000 \mu \mathrm{l}$ washing buffer, homogenize and keep on ice for $10 \mathrm{~min}$; (4) Centrifuge the tube at $12,000 \times g$ for $10 \mathrm{~min}$ at $4^{\circ} \mathrm{C}$; (5) Remove and discard the supernatant, and then repeat steps $3-$ 5; (6) Add $1000 \mu \mathrm{l} \mathrm{CTAB} \mathrm{buffer,} \mathrm{mix} \mathrm{and} \mathrm{incubate} \mathrm{at} 65^{\circ} \mathrm{C}$ for $3 \mathrm{~h}$ with occasional mixing; (7) Centrifuge at 12,000 rpm for $10 \mathrm{~min}$ at $4^{\circ} \mathrm{C}$, transfer supernatant to a new $2 \mathrm{ml}$ tube; (8) Add an equal volume of extraction buffer to the tube and mix by inversion for $5 \mathrm{~min}$; (9) Centrifuge at $12,000 \mathrm{rpm}$ for $10 \mathrm{~min}$ at $4^{\circ} \mathrm{C}$, transfer the top layer into a fresh tube; (10) Repeat steps 8-9; (11) Add an equal volume of cold absolute isopropanol and incubate for $1 \mathrm{~h}$ at $-20^{\circ} \mathrm{C}$; (12) Transfer the mixture into a spin column (Sangon, Shanghai, China) placed in a $2 \mathrm{ml}$ collection tube, add $500 \mu \mathrm{l}$ cold $70 \%$ ethanol, and centrifuge for $1 \mathrm{~min}$ at $10,000 \mathrm{rpm}$, discard the flow-through; (13) Add $500 \mu \mathrm{l}$ absolute cold 100\% ethanol, centrifuge for $3 \mathrm{~min}$ at 10,000 rpm; (14) Transfer the spin column

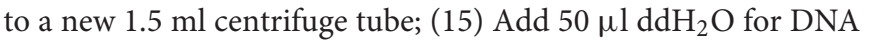
elution. The key points to consider in the DNA extraction of herb materials are summarized as following: (1) Because the herbal materials are usually harvested long before they are used, the surface of herbal material should be cleaned with $75 \%$ alcohol prior to being ground to a fine powder in liquid nitrogen for DNA extraction; (2) The high levels of polysaccharides and polyphenols in some herbal materials must be removed using polyvinylpyrrolidone (PVP) and $\beta$-mercaptoethanol during the early stages of DNA extraction; (3) In most cases, medicinal plant products are commonly sold in processed or modified forms such as dried material, tablets or powders, making its DNA degraded severely; thus, the dosage of plant starting material, PVP, and $\beta$-mercaptoethanol must be increased accordingly for DNA extraction.

\section{Optimization}

For successful analysis, the experimental conditions should be optimized for Bar-HRM. Small differences in melting curves can arise from factors such as the DNA quality and quantity, amplicon length, primer design, and cycling conditions. Optimize details was discussed above, under the Section "Key Factors Affecting HRM Assays." Here, we summarize the key points as following: (1) All DNA samples should be extracted using the same DNA extraction method; the DNA sample should be diluted to a similar concentration in the same buffer. (2) The primer must be specific enough, and an optimal concentration is also required. (3) Adjustment of the annealing/extension time. Because shorter times result in incomplete amplicons, longer times can increase the possibility of mispriming and non-specific amplification. (4) The melting temperature should be adjusted to 
appropriate range according to the characteristic of the amplified products.

\section{HRM-PCR Amplification}

In theory, all of the plant DNA barcoding regions (i.e., ITS, $p s b \mathrm{~A}$ $\operatorname{trnH}, r b c \mathrm{~L}, m a t \mathrm{~K}, r p o \mathrm{C}, t r n \mathrm{~L}$, etc.) can used as barcodes for identifying herbal materials through Bar-HRM. For reference, we list the universal primers for the ITS2 barcode (S2F: $5^{\prime}$-ATG CGA TAC TTG GTG TGA AT-3'; S3R: 5'-GAC GCT TCT CCA GAC TAC AAT- $3^{\prime}$ ) and the $p s b$ A-trnH barcode (PA: $5^{\prime}$-GTT ATG CAT GAA CGT ATG CTC-3' ${ }^{\prime}$; TH: $5^{\prime}$-CGC GCA TGG TGG ATT CAC AAT CC-3'; Chen et al., 2010). In some cases, mini-barcode primers have been designed to distinguish samples from closely related species (Osathanunkul et al., 2015a,b; Costa et al., 2016). It is recommended that real-time PCR be conducted; amplification can then be monitored, and any troubleshooting that may be required is made easier. The reaction conditions and components used for PCR amplification can be found in the respective studies (Kalivas et al., 2014; Tong et al., 2014; Buddhachat et al., 2015; Hu et al., 2015; Osathanunkul et al., 2015a,b; Singtonat and Osathanunkul, 2015; Costa et al., 2016).

\section{Melting Profile Analysis, Species Identification, and Adulteration Detection}

Basic analysis of melting profile is described above, under the Section "HRM Data Analysis." Difference curves, which accentuate the differences between individual melt curves, have been used to identify species. Test samples were defined by selecting an original representative sample for each species, and then a single reference-species melt curve was plotted as a baseline, providing improved visualization and allowing the separation of the melting curves of each species. Regardless of which species was used as the reference genotype, the position and shape of the difference curves of the other samples consistently allowed species identification (Kalivas et al., 2014). To detect the amount of adulterant in samples, standard purity curves were established by plotting the fluorescence level against

\section{REFERENCES}

Akiyoshi, K., Yamada, Y., Honma, Y., Iwasa, S., Kato, K., Hamaguchi, T., et al. (2013). KRAS mutations in patients with colorectal cancer as detected by high-resolution melting analysis and direct sequencing. Anticancer Res. 33, 2129-2134.

Bosmali, I., Ganopoulos, I., Madesis, P., and Tsaftaris, A. (2012). Microsatellite and DNA-barcode regions typing combined with High Resolution Melting (HRM) analysis for food forensic uses: a case study on lentils (Lens culinaris). Food Res. Int. 46, 141-147. doi: 10.1016/j.foodres.2011. 12.013

Bridson, D. M., and Forman, L. (1992). The Herbarium Handbook. London: Royal Botanic Gardens Kew.

Buddhachat, K., Osathanunkul, M., Madesis, P., Chomdej, S., and Ongchai, S. (2015). Authenticity analyses of Phyllanthus amarus using barcoding coupled with HRM analysis to control its quality for medicinal plant product. Gene 573, 84-90. doi: 10.1016/j.gene.2015. 07.046

Chase, M. W., Salamin, N., Wilkinson, M., Dunwell, J. M., Kesanakurthi, R. P., Haidar, N., et al. (2005). Land plants and DNA barcodes: short-term and long-term goals. Philos. Trans. R. Soc. Lond. B. Biol. Sci. 360, 1889-1895. doi: $10.1098 /$ rstb.2005.1720 the percentage of the adulterant (Buddhachat et al., 2015; Singtonat and Osathanunkul, 2015); the shape of the HRM curves indicates if the herbal product contains one or more adulterants.

\section{CONCLUSION}

In recent years, the identification of species in herbal products has gained increased attention due to concerns about quality control and the satisfaction and safety of consumers. Recent advancements in molecular biology have led to the development of various criteria and methods to tackle this problem, one of the most remarkable being the rapid development of Bar-HRM technology. Although the application of Bar-HRM analysis in the authentication of herbal products is still in its initial stages, it shows great potential for identifying and tracking a wide range of raw and processed herbal medicine products, as well as detecting adulteration or poisonous contaminants in herbal products. With continued development and improvement, the Bar-HRM assay will become a significant resource for accurate species identification, monitoring, and quality control of herbal and other medicinal materials.

\section{AUTHOR CONTRIBUTIONS}

WS and J-jL wrote the manuscript. CX help to collect references articles. BZ help with language editing support. S-lC provided helpful comments on the article.

\section{ACKNOWLEDGMENTS}

This work was funded by the National Natural Science Foundation of China (No. 81130069 and No. 81373922) and the Fundamental Research Funds for the Central Scientific Research Institutes for Public Welfare (No. YZ-12-08).

Chen, C. F., Chou, W. F., and Zhang, J. T. (2008). Comparison of the pharmacological effects of Panax ginseng and Panax quinquefolium. Acta Pharmacol. Sin. 29, 1103-1108. doi: 10.1111/j.1745-7254.2008.00868.x

Chen, L. Y., Song, M. S., and Zha, H. G. (2014). A modified protocol for plant genome DNA extraction. Plant Divers Resour. 36, 375-380.

Chen, S., Yao, H., Han, J., Liu, C., Song, J., Shi, L., et al. (2010). Validation of the ITS2 region as a novel DNA barcode for identifying medicinal plant species. PLoS ONE 5:e8613. doi: 10.1371/journal.pone.0008613

Chen, S. L., Yao, H., Han, J. P., Xin, T. Y., Pang, X. H., Shi, L. C., et al. (2013). Principles for molecular identification of traditional Chinese meteria medica using DNA barcoding. China J. Chin. Mater. Med. 38, 141-148.

Consortium for the Barcode of Life (CBOL) Plant Working Group (2009). A DNA barcode for land plants. Proc. Natl. Acad. Sci. U.S.A. 106, 12794-12797. doi: 10.1073/pnas.0905845106

Costa, J., Campos, B., Amaral, J. S., Nunesc, M. E., Beatriz, M., Oliveira, P. P., et al. (2016). HRM analysis targeting ITS1 and matK loci as potential DNA mini-barcodes for the authentication of Hypericum perforatum and Hypericum androsaemum in herbal infusions. Food Control 6, 105-114. doi: 10.1016/j.foodcont.2015.09.035

Debelle, F. D., Vanherweghem, J. L., and Nortier, J. L. (2008). Aristolochic acid nephropathy: a worldwide problem. Kidney Int. 74, 158-169. doi: 10.1038/ki.2008.129 
Dunning, L. T., and Savolainen, V. (2010). Broad-scale amplification of matK for DNA barcoding plants, a technical note. Bot. J. Linn. Soc. 164, 1-9. doi: $10.1111 / \mathrm{j} .1095-8339.2010 .01071 . \mathrm{x}$

Duyvejonck, H., Cools, P., Decruyenaere, J., Roelens, K., Noens, L., Vermeulen, S., et al. (2015). Validation of high resolution melting analysis (HRM) of the amplified ITS2 region for the detection and identification of yeasts from clinical samples: comparison with culture and MALDI-TOF based identification. PLoS ONE 10:e0132149. doi: 10.1371/journal.pone.0132149

Erali, M., Voelkerding, K. V., and Wittwer, C. T. (2008). High resolution melting applications for clinical laboratory medicine. Exp. Mol. Pathol. 85, 50-58. doi: 10.1016/j.yexmp.2008.03.012

Fazekas, A. J., Burgess, K. S., Kesanakurti, P. R., Graham, S. W., Newmaster, S. G., Husband, B. C., et al. (2008). Multiple multilocus DNA barcodes from the plastid genome discriminate plant species equally well. PLoS ONE 3:e2802. doi: 10.1371/journal.pone.0002802

Galimberti, A., Mattia, F. D., Losa, A., Brunai, I., Federici, S., Casiraghi, M., et al. (2013). DNA barcoding as a new tool for food traceability. Food Res. Int. 50, $55-63$.

Galimberti, A., Romano, D. F., Genchi, M., Paoloni, D., Vercillo, F., Bizzarri, L., et al. (2012). Integrative taxonomy at work: DNA barcoding of taeniids harboured by wild and domestic cats. Mol. Ecol. Resour. 12, 403-413. doi: 10.1111/j.1755-0998.2011.03110.x

Ganopoulos, I., Madesis, P., Darzentas, N., Argiriou, A., and Tsaftaris, A. (2012). Barcode High Resolution Melting (Bar-HRM) analysis for detection and quantification of PDO "Fava Santorinis" (Lathyrus clymenum) adulterants. Food Chem. 133, 505-512. doi: 10.1016/j.foodchem.2012.01.015

GIA (2013). Herbal Supplements and Remedies - A Global Strategic Business Report. San Jose, CA: Global Industry Analysts Inc.

Graham, R., Liew, M., Lyon, E., Lyon, E., and Wittwer, C. T. (2005). Distinguishing different DNA heterozygotes by high-resolution melting. Clin. Chem. 51, 1295-1298. doi: 10.1373/clinchem.2005.051516

Grollman, A. P., Shibutani, S., Moriya, M., Miller, F., Wu, L., Moll, U., et al. (2007). Aristolochic acid and the etiology of endemic (Balkan) nephropathy. Proc. Natl. Acad. Sci. U.S.A. 104, 12129-12134. doi: 10.1073/pnas.0701248104

Hebert, P. D., Cywinska, A., Ball, S. L., and deWaard, J. R. (2003). Biological identifications through DNA barcodes. Proc. R. Soc. Biol. Sci. Ser. B 270, 313-321. doi: 10.1098/rspb.2002.2218

Hebert, P. D. N., Stoeckle, M. Y., Zemlak, T. S., and Francis, C. M. (2004). Identification of birds through DNA barcodes. PLoS Biol. 2:e312. doi: 10.1371/journal.pbio.0020312

Herrmann, M. G., Durtschi, J. D., Bromley, L. K., Wittwer, C. T., and Voelkerding, K. V. (2006). Amplicon DNA melting analysis for mutation scanning and genotyping: cross-platform comparison of instruments and dyes. Clin. Chem. 52, 494-503. doi: 10.1373/clinchem.2005.063438

Hu, J., Zhan, Z. L., Yuan, Y., Huang, L. Q., and Liu, Y. (2015). HRM identification of Chinese medicinal materials Mutong. China J. Chin. Mater. Med. 40, 2304-2308.

Kalivas, A., Ganopoulos, I., Xanthopoulou, A., Chatzopoulou, P., Tsaftaris, A., and Madesis, P. (2014). DNA barcode ITS2 coupled with high resolution melting (HRM) analysis for taxonomic identification of Sideritis species growing in Greece. Mol. Biol. Rep. 41, 5147-5155. doi: 10.1007/s11033-014-3381-5

Kress, W. J., and Erickson, D. L. (2007). A two-locus global DNA barcode for land plants: the coding rbcL gene complements the non-coding trnH-psbA spacer region. PLOS ONE 2:e508. doi: 10.1371/journal.pone.0000508

Kress, W. J., Wurdack, K. J., Zimmer, E. A., Weigt, L. A., and Janzen, D. H. (2005). Use of DNA barcodes to identify flowering plants. Proc. Natl. Acad. Sci. U.S.A. 102, 8369-8374. doi: 10.1073/pnas.0503123102

Krypuy, M., Newnham, G. M., Thomas, D. M., Conron, M., and Dobrovic, A. (2006). High resolution melting analysis for the rapid and sensitive detection of mutations in clinical samples: KRAS codon 12 and 13 mutations in non-small cell lung cancer. BMC Cancer 6:295. doi: 10.1186/1471-2407-6-295

Lau, C. B., Cheng, L., Cheng, B. W., Yue, G. G., Wong, E. C., Lau, C. P., et al. (2012). Development of a simple chromatographic method for distinguishing between two easily confused species, Hedyotis diffusa and Hedyotis corymbosa. Nat. Prod. Res. 26, 1446-1450. doi: 10.1080/14786419.2011.603317

Li, J., Men, Q. M., and Liu, J. P. (2012). Research on Hyoscyamus niger. Chin. Arch. Tradit. Chin. Med. 30, 615-618.
Li, M., Cao, H., But, P. P. H., and Shaw, P. C. (2011). Identification of herbal medicinal materials using DNA barcodes. J. Syst. Evol. 49, 271-283. doi: 10.1111/j.1759-6831.2011.00132.x

Li, M., Jiang, R. W., Hon, P. M., Cheng, L., Li, L. L., Zhou, J. R., et al. (2010). Authentication of the anti-tumor herb Baihuasheshecao with bioactive marker compounds and molecular sequences. Food Chem. 119, 1239-1245. doi: 10.1016/j.foodchem.2009.09.013

Lin, C. Y., Li, M. J., Chang, J. G., Liu, S. C., Weng, T., Wu, K. H., et al. (2014). Highresolution melting analyses for genetic variants in ARID5B and IKZF1 with childhood acute lymphoblastic leukemia susceptibility loci in Taiwan. Blood Cells Mol. Dis. 52, 140-145. doi: 10.1016/j.bcmd.2013.10.003

Liu, C., Liang, D., Gao, T., Pang, X., Song, J., Yao, H., et al. (2011). PTIGS-IdIt, a system for species identification by DNA sequences of the psbA-trnH intergeic spacer region. BMC Bioinformatics 12:S4. doi: 10.1186/1471-2105-12-S13-S4

Lord, G. M., Tagore, R., Cook, T., Gower, P., and Pusey, C. D. (1999). Nephropathy caused by Chinese herbs in the UK. Lancet 354, 481-482. doi: 10.1016/S01406736(99)03380-2

Mader, E., Ruzicka, J., Schmiderer, C., and Novak, J. (2011). Quantitative highresolution melting analysis for detecting adulterations. Anal. Biochem. 409, 153-155. doi: 10.1016/j.ab.2010.10.009

Madesis, P., Ganopoulos, I., Anagnostis, A., and Tsaftaris, A. (2012). The application of Bar-HRM (Barcode DNA-High Resolution Melting) analysis for authenticity testing and quantitative detection of bean crops (Leguminosae) without prior DNA purification. Food Control 25, 576-582. doi: 10.1016/j.foodcont.2011.11.034

Mahadani, P., and Ghosh, S. K. (2013). DNA Barcoding: a tool for species identification from herbal juices. DNA Barcodes 1, 35-38. doi: 10.2478/dna2013-0002

Miller, S. E. (2007). DNA barcoding and the renaissance of taxonomy. Proc. Natl. Acad. Sci. U.S.A. 104, 4775-4776. doi: 10.1073/pnas.0700466104

Montgomery, J., Wittwer, C. T., Palais, R., and Zhou, L. (2007). Simultaneous mutation scanning and genotyping by high-resolution DNA melting analysis. Nat. Protoc. 2, 59-66. doi: 10.1038/nprot.2007.10

Montgomery, J. L., Sanford, L. N., and Wittwer, C. T. (2010). High-resolution DNA melting analysis in clinical research and diagnostics. Expert Rev. Mol. Diagn. 10, 219-240. doi: 10.1586/erm.09.84

Mower, J. P., Touzet, P., Gummow, J. S., Delph, L. F., and Palmer, J. D. (2007). Extensive variation in synonymous substitution rates in mitochondrial genes of seed plants. BMC Evol. Biol. 7:135. doi: 10.1186/1471-2148-7-135

Nair, R. R., Udayan, P. S., Thilaga, S., Kavitha, M., Bharathanandhini, R. M., Nizzy, A. M., et al. (2013). Molecular distinction of two closely resembling Morinda species using $\mathrm{rbcL}$ and matK loci for quality management of Indian herbal medicines. Plant Genet. Resour. 11, 90-93. doi: 10.1017/S147926211200041X

Newmaster, S. G., Grguric, M., Shanmughanandhan, D., Ramalingam, S., and Ragupathy, S. (2013). DNA barcoding detects contamination and substitution in North American herbal products. BMC Med. 11:222. doi: 10.1186/17417015-11-222

Osathanunkul, M., Madesis, P., and Boer, H. D. (2015a). Bar-HRM for authentication of plant-based medicines: evaluation of three medicinal products derived from Acanthaceae species. PLOS ONE 10:e0128476. doi: 10.1371/journal.pone.0128476

Osathanunkul, M., Suwannapoom, C., Ounjai, S., Rora, J. A., Madesis, P., and de, Boer H (2015b). Refining DNA barcoding coupled high resolution melting for discrimination of 12 closely related croton species. PLOS ONE 10:e138888. doi: 10.1371/journal.pone.0138888

Ouarghidi, A., Powell, B., Martin, G. J., Boer, H. De, and Abbad, A. (2012). Species substitution in medicinal roots and possible implications for toxicity in Morocco. Econ. Bot. 66, 370-382. doi: 10.1007/s12231-012-9 215-2

Palais, R., Liew, M., and Wittwer, C. (2005). Quantitative heteroduplex analysis for single nucleotide polymorphism genotyping. Anal. Biochem. 346, 167-175. doi: 10.1016/j.ab.2005.08.010

Pang, X., Shi, L., Song, J., Chen, X., and Chen, S. (2013). Use of the potential DNA barcode ITS2 to identify herbal materials. J. Nat. Med. 67, 571-575. doi: 10.1007/s11418-012-0715-2

Poon, S. L., Pang, S. T., McPherson, J. R., Yu, W., Huang, K. K., Guan, P., et al. (2013). Genome-wide mutational signatures of aristolochic acid and 
its application as a screening tool. Sci. Transl. Med. 5:197ra101. doi: 10.1126/scitranslmed.3006086

Reed, G. H., Kent, J. O., and Wittwer, C. T. (2007). High-resolution DNA melting analysis for simple and efficient molecular diagnostics. Pharmacogenomics 8, 597-608.

Reed, G. H., and Wittwer, C. T. (2004). Sensitivity and specificity of singlenucleotide polymorphism scanning by high-resolution melting analysis. Clin. Chem. 50, 1748-1754. doi: 10.1373/clinchem.2003.029751

Ririe, K. M., Rasmussen, R. P., and Wittwer, C. T. (1997). Product differentiation by analysis of DNA melting curves during the polymerase chain reaction. Anal. Biochem. 245, 154-160. doi: 10.1006/abio.1996.9916

Ryan, G. T. (2005). DNA barcoding does not compete with taxonomy. Nature 434, 1067-1067. doi: 10.1038/4341067b

Sakaridis, I., Ganopoulos, I., Argiriou, A., and Tsaftaris, A. (2013). A fast and accurate method for controlling the correct labeling of products containing buffalo meat using High Resolution Melting (HRM) analysis. Meat Sci. 94, 84-88. doi: 10.1016/j.meatsci.2012.12.017

Sass, C., Little, D. P., Stevenson, D. W., and Specht, C. D. (2007). DNA barcoding in the Cycadales: testing the potential of proposed barcoding markers for species identification of Cycads. PLoS ONE 2:e1154. doi: 10.1371/journal.pone.0001154

Shen, Y., Li, Y., and Liu, T. T. (2014). Microscopical and physicochemcical identification of 3 sources of Curcumae Rhizoma. Chem. Res. Appl. 26, 267-270.

Singtonat, S., and Osathanunkul, M. (2015). Fast and reliable detection of toxic Crotalaria spectabilis Roth. in Thunbergia laurifolia Lindl. herbal products using DNA barcoding coupled with HRM analysis. BMC Complement. Altern. Med. 15:162. doi: 10.1186/s12906-015-0692-6

Sun, Z., Gao, T., Yao, H., Shi, L., Zhu, Y., and Chen, S. (2011). Identification of Lonicera japonica and its related species using the DNA barcoding method. Planta Med. 77, 301-306. doi: 10.1055/s-0030-1250324

Tel-zur, N., Abbo, S., Myslabodski, D., and Mizrahi, Y. (1999). Modified CTAB procedure for DNA isolation from epiphytic cacti of the genera Hylocereus and Selenicereus (Cactaceae). Plant Mol. Biol. Rep. 17, 249-254. doi: 10.1023/A:1007656315275

Tong, Y. R., Jiang, C., Huang, L. Q., Cui, Z. H., and Yuan, Y. (2014). Molecular identification of Radix Notoginseng powder by DNA melt curve analysis. Chin. J. Pharm. Anal. 34, 1384-1390.

Tu, Y., Xiong, C., Shi, Y. H., Ming, M., Hu, Z., and Huang, B. (2014). Identification of toxic hyoscyami semen and its adulterants using DNA barcoding. World Sci. Technol. Mod. Tradit. Chin. Med. Mater. Med. 16, 2337-2342.

van der Stoep, N., van Paridon, C. D., Janssens, T., Krenkova, P., Stambergova, A., Macek, M., et al. (2009). Diagnostic guidelines for high-resolution melting curve (HRM) analysis: an interlaboratory validation of BRCA1 mutation scanning using the 96-well lightscanner. Hum. Mutat. 30, 899-909. doi: 10.1002/humu. 21004

Vanherweghem, J. L., Depierreux, M., Tielemans, C., Abramowicz, D., Dratwa, M., Jadoul, M., et al. (1993). Rapidly progressive interstitial renal fibrosis in young women: association with slimming regimen including Chinese herbs. Lancet 341, 387-391. doi: 10.1016/0140-6736(93)92984-2

Veldman, S., Otieno, J., Gravendeel, B., van Andel, T., and de Boer, H. (2014). "Conservation of endangered wild harvested medicinal plants: use of DNA barcoding," in Novel Plant Bioresources: Applications in Food, Medicine and Cosmetics, ed. A. Gurib-Fakim (Chichester: John Wiley \& Sons, Ltd), 81-88. doi: 10.1002/9781118460566.ch6

von Ahsen, N., Oellerich, M., and Schutz, E. (2001). Limitations of genotyping based on amplicon melting temperature. Clin. Chem. 47, 1331-1332.
Vossen, R. H., Aten, E., Roos, A., and den Dunnen, J. T., (2009). Highresolutionmelting analysis (HRMA) - More than just sequence variant screening. Hum. Mutat. 30, 860-866. doi: 10.1002/humu.21019

Ward, R. D., Zemlak, T. S., Innes, B. H., Last, P. R., and Hebert, P. D. (2005). DNA barcoding Australia's fish species. Philos. Trans. R. Soc. Lond. B Biol. Sci. 360, 1847-1857. doi: 10.1098/rstb.2005.1716

Wittwer, C. T. (2009). High-resolution DNA melting analysis: advancements and limitations. Hum. Mutat. 30, 857-859. doi: 10.1002/humu.20951

Wittwer, C. T., Herrmann, M. G., Moss, A. A., and Rasmussen, R. P. (1997). Continuous fluorescence monitoring of rapid cycle DNA amplification. Biotechniques 22, 134-138.

Wittwer, C. T., Reed, G. H., Gundry, C. N., Vandersteen, J. G., and Pryor, R. J. (2003). High-resolution genotyping by amplicon melting analysis using LC Green. Clin. Chem. 49, 853-860. doi: 10.1373/49.6.853

Wojdacz, T. K. (2012). Methylation-sensitive high-resolution melting in the context of legislative requirements for validation of analytical procedures for diagnostic applications. Expert Rev. Mol. Diagn. 12, 39-47. doi: 10.1586/ERM.11.88

Wojdacz, T. K., Windeløv, J. A., Thestrup, B. B., Damsgaard, T. E., Overgaard, J., and Hansen, L. (2014). Identification and characterization of locus-specific methylation patterns within novel loci undergoing hypermethylation during breast cancer pathogenesis. Breast Cancer Res. 16:R17. doi: 10.1186/b cr3612

World Health Organization [WHO] (2003). Traditional Medicine. Factsheet No. 134. Available at: http://www.who.int/mediacentre/news/releases/2004/ pr44/en/

World Health Organization (2013). WHO Traditional Medicine Strategy, 20142023. Hong Kong: World Health Organization.

Wu, L., Sun, W., Wang, B., Zhao, H., Li, Y., Cai, S., et al. (2015). An intergrated system for identifying the hidden assassins in traditional medicines containing aristolochic acids. Sci. Rep. 5:1138. doi: 10.1038/srep11318

Xanthopoulou, A., Ganopoulos, I., Kalivas, A., and Osathanunkul, M. (2016). Multiplex HRM analysis as a tool for rapid molecular authentication of nine herbal teas. Food Control 60, 113-116. doi: 10.1016/j.foodcont.2015.07.021

Xin, T., Li, X., Yao, H., Lin, Y., Ma, X., Cheng, R., et al. (2015). Survey of commercial Rhodiola products revealed species diversity and potential safety issues. Sci. Rep. 5:8337. doi: 10.1038/srep08337

Zhou, L., Wang, L., Palais, R., Pryor, R., and Wittwer, C. T. (2005). High-resolution DNA melting analysis for simultaneous mutation scanning and genotyping in solution. Clin. Chem. 51, 1770-1777. doi: 10.1373/clinchem.2005.054924

Zomuanpuii, R., Ringngheti, L., Brindha, S., Gurusubramanian, G., and Senthil Kumar, N. (2013). ITS2 characterization and Anopheles species identification of the subgenus Cellia. Acta Trop. 125, 309-319. doi: 10.1016/j.actatropica.2012.12.001

Conflict of Interest Statement: The authors declare that the research was conducted in the absence of any commercial or financial relationships that could be construed as a potential conflict of interest.

Copyright (c) 2016 Sun, Li, Xiong, Zhao and Chen. This is an open-access article distributed under the terms of the Creative Commons Attribution License (CC BY). The use, distribution or reproduction in other forums is permitted, provided the original author(s) or licensor are credited and that the original publication in this journal is cited, in accordance with accepted academic practice. No use, distribution or reproduction is permitted which does not comply with these terms. 